\title{
PICALM rs3851179 Variant and Alzheimer's Disease in Asian Population
}

\author{
Yining $\mathrm{Xu}^{1} \cdot$ Qinghua Jiang ${ }^{2} \cdot$ Guiyou Liu $^{2}$
}

Received: 23 February 2016/Accepted: 5 March 2016/Published online: 17 March 2016

(C) Springer Science+Business Media New York 2016

To the editor

We thank Wang and colleagues for their interest in our article published in NeuroMolecular Medicine (Liu et al. 2013). In this article above, we evaluated the potential association of rs3851179 variant with Alzheimer's disease (AD) in Asian population by a pooled analysis and a metaanalysis. The results show that rs3851179 variant is significantly associated with AD in Asian population by both methods. Here, Wang and colleagues discussed three issues.

First, Wang and colleagues described that an exhaustive search is necessary for meta-analysis. We searched the PubMed database to select all the possible studies in the original article (Liu et al. 2013). We accept this suggestion and will use multiple literature databases in further studies, including PubMed, AlzGene, Google Scholar and China Knowledge Resource Integrated Database (CNKI), as did in our recent studies (Liu et al. 2014; Shen et al. 2015; Li et al. 2015; Zhang et al. 2015).

Second, we thank Wang and colleagues again, as they pointed out a writing error about the significance level of Cochran's $Q$ test $(0.01$ and 0.05$)$ in the "Evaluation of the Heterogeneity" section. We also accept the suggestion about the Cochran's $Q$ test significance level 0.1. In further, we will give an erratum to this article published in NeuroMolecular Medicine (Liu et al. 2013).

Third, we accept the suggestion that the Hardy-Weinberg equilibrium (HWE) test should be conducted only in

Guiyou Liu

liuguiyou1981@163.com

1 School of Computer Science and Technology, Harbin Institute of Technology, Harbin, China

2 School of Life Science and Technology, Harbin Institute of Technology, Room 415, Building 2E, Science Park, Yikuang Street, Nangang District, Harbin 150080, China controls but no in cases in case-control studies. We think it may not suitable for combining the data of China and Japan. In further study, we will calculate the $p$ value for HWE, respectively.

In summary, we think that these three issues are important, and may provide supplementary information about our previous article (Liu et al. 2013).

Acknowledgments This work was supported by funding from the National Nature Science Foundation of China (Grant Nos. 81300945 and 61571152) and National Science and Technology Major Project of China (Grant No. 2012ZX09304003).

\section{Compliance with Ethical Standards}

Conflict of interests The authors declare no competing financial interests.

\section{References}

Li, X., Shen, N., Zhang, S., Liu, J., Jiang, Q., Liao, M., et al. (2015). CD33 rs3865444 polymorphism contributes to Alzheimer's disease susceptibility in Chinese, European, and North American populations. Molecular Neurobiology, 52(1), 414-421.

Liu, G., Li, F., Zhang, S., Jiang, Y., Ma, G., Shang, H., et al. (2014). Analyzing large-scale samples confirms the association between the ABCA7 rs3764650 polymorphism and Alzheimer's disease susceptibility. Molecular Neurobiology, 50(3), 757-764.

Liu, G., Zhang, S., Cai, Z., Ma, G., Zhang, L., Jiang, Y., et al. (2013). PICALM gene rs 3851179 polymorphism contributes to Alzheimer's disease in an Asian population. Neuromolecular Medicine, 15(2), 384-388.

Shen, N., Chen, B., Jiang, Y., Feng, R., Liao, M., Zhang, L., et al. (2015). An updated analysis with 85,939 samples confirms the association between CR1 rs6656401 polymorphism and Alzheimer's disease. Molecular Neurobiology, 51(3), 1017-1023.

Zhang, S., Li, X., Ma, G., Jiang, Y., Liao, M., Feng, R., et al. (2015). CLU rs9331888 polymorphism contributes to Alzheimer's disease susceptibility in Caucasian but not East Asian populations. Molecular Neurobiology, 30, 1-6. 